\title{
Caracterización
}

\section{de la arquitectura vernácula en madera de complejos constructivos rurales, región de Aysén, Chile}

Characterization of the Vernacular Wooden Architecture from Rural Building Complexes, Aysén Region, Chile

\author{
Carlos Castillo Levicoy \\ Investigador independiente, Chile \\ castillolevicoycarlos@gmail.com

\section{Constanza Pérez Lira} \\ Investigadora independiente, Chile \\ constanzaperezlira@gmail.com
}

\section{Resumen}

Este INFORME presenta los resultados tras medir las construcciones de arquitectura vernácula en madera de complejos constructivos rurales en la región de Aysén, Chile, en planta y elevación; además se identifica su materialidad constructiva y escuadrías y determinar el año de asentamiento. La construcción más temprana corresponde a El Fraile, 1926 y 1932: Lago Largo, 1930-1932; Cerro La Virgen, 1932 y 1933; Lago Cástor, 1935; El Salto, 1935, y Seis Lagunas, 1936. Los revestimientos corresponden a la tejuela artesanal, tabla vertical con cinta tapa-junta, tabla horizontal traslapada (tingladillo), machihembrado y el tablón labrado-amordazado y estopado. Los tipos de cubierta de "un agua, dos aguas y cuatro aguas", la volumetría simple "rectangular" y compuesta en forma de $L$ y $U$ fue la más común que se observa en los complejos constructivos.

\section{Palabras clave}

arquitectura vernácula; madera; sector rural; región de Aysén, Chile

\section{Abstract}

This REPORT presents the results obtained from the measurement, both in plan and elevation, of the vernacular wooden architecure from rural building complexes in the Aysén región in Chile. Furthermore, it identifies their constructive materiality, scantling, and determines the year of settlement. The earliest construction corresponds to El Fraile, 1926 and 1932; Lago Largo, 1930-1932; Cerro La Virgen, 1932 and 1933; Lago Cástor, 1935; El Salto, 1935; and Seis Lagunas, 1936. The 
facings correspond to hand-crafted shingles, a vertical table with a joint cover strip, overlapping horizontal boards (clinker), tongue and groove joint, and a shiplap joint and oakum caulking. The "shed, gable, and hip" roof types, and the simple "rectangular" volumetry in $L$ and $U$ shapes were the most commonly observed in building complexes.

\section{Keywords}

vernacular architecture; wood; rural sector; Aysén region; Chile

\section{Introducción}

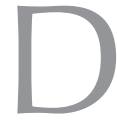
urante los primeros años del siglo $\mathrm{XX}$, la estrategia utilizada por el Estado chileno para hacer efectiva la ocupación del territorio de Aysén se basó en la concesión de tierras fiscales a empresas pecuarias, de las cuales sólo tres sobrevivieron: la Compañía Ganadera de Río Cisnes, la Compañía Explotadora del Baker y la Sociedad Industrial de Aisén (SIA) (Osorio 2014: 52). Se les exigió el cumplimiento de ciertos compromisos, como crear infraestructura adecuada, activar la productividad del territorio y, especialmente, fomentar nuevos asentamientos de pobladores que contribuirían a la ocupación e integración de la zona al resto del territorio nacional (Martinic 2016: 244-308; Osorio 2014: 52-75). Sin embargo, esto no se satisfizo. El poblamiento definitivo del territorio de Aysén se desarrolló por medio de una colonización espontánea y autogestionada. Los pobladores, junto con sus familias, se adentraron en el territorio vía litoral, y por la zona limítrofe con Argentina comenzaron a construir, de forma muy precaria, los primeros ranchos o puestos, utilizando los conocimientos y técnicas aprendidos en sus lugares de origen y con las escasas herramientas disponibles. A medida que los campos se limpiaban y habilitaban para la crianza de animales y las siembras, se construían viviendas más sólidas y de mayores comodidades. Esa arquitectura evolucionó en forma paralela al proceso de poblamiento y ocupación de la región de Aysén, Chile, a lo largo del siglo XX, pasando desde una arquitectura de emergencia o de "casas refugio" —que cumplían con las condiciones mínimas para la subsistencia—, hasta una "casa definitiva", donde el perfeccionamiento en el trabajo de la madera y la incorporación de nuevas tecnologías serían representativos del carácter más estable que adquirieron los asentamientos llevados a cabo por los nuevos colonos (Pérez et al. 2018: 35; Castillo 2015: 18).

Con presencia más notoria y estable dentro del territorio aysenino por parte del Estado y sus instituciones, las condiciones de habitabilidad mejoraron marcadamente y la arquitectura comenzó a tener un carácter más definitivo (Pérez et al. 2018: 34). Un factor que incidió en la mejora de las técnicas constructivas fue la instalación, a mediados del siglo XX, de aserraderos, con cuya maquinaria se multiplicó la producción y se hicieron acabados más elaborados y precisos, mejorando el diseño de las construcciones y perfeccionando aquéllos en sus interiores, tanto en muros y pisos como en puertas y ventanas (Castillo 2015a: 18). A la mecanización de la producción maderera hay que sumar la utilización de nuevas herramientas para la construcción en madera (sierras, máquinas cepilladoras y machihembradoras, cepillos manuales, taladros, etc.), las que complementaron el trabajo que realizaban aquellas herramientas más básicas, como el hacha. Otro factor fundamental que permitió un mayor desarroIlo de la arquitectura habitacional fue la llegada de mano de obra especializada. Con las corrientes migratorias que arribaron a la región desde otros sectores del sur del país y las zonas fronterizas argentinas, Ilegaron oficios tradicionales: madereros, carpinteros, tejueleros y mueblistas, entre otros. Especialmente significativo fue el acervo cultural proveniente de carpinteros chilotes, cuyos conocimientos, habilidades $-y$ también sus herramientas para trabajar la madera- se reflejarían en los sistemas constructivos y la arquitectura más propia de Aysén (Bozzolo 1992). Cabe destacar que en esa región, la presencia y la disponibilidad de elementos como la madera, piedras, arcillas, entre otros, posibilitaron la construcción de cobijos rústicos difíciles de evidenciar en la actualidad, destacando el uso del junquillo o canutillo, las canogas o casqueros y palos partidos, de palos o tablones labrados y amordazadosestopados, ${ }^{1}$ de pared francesa ${ }^{2}$ y de piedras (García 2000: 344-345; Castillo 2015a: 18). Otros cobijos más elaborados permanecen y testimonian hoy las distintas corrientes migratorias de pioneros eurochilenos que se adentraron y asentaron en territorio aysenino, donde destaca el ladrillo cocido, el adobe, las maderas labradas a hacha y aserradas tanto en borriquetes como en aserraderos más convencionales, unidas con clavos de madera $(\operatorname{tarugos})^{3}$ y con clavos de hierro, con revestimiento de tejuela artesanal, tablas horizontales traslapadas (tingladillo), tablas verticales con cintas tapajuntas ${ }^{4}$ y el zinc (Castillo 2015: 18; Pérez y Castillo 2016: 9; Pérez et al. 2018: 60-149).

Esas construcciones, que datan de principios y mediados del siglo XX, se concentran mayoritariamente en los sectores rurales alejados de las localidades urbanas. Casas, cocinas-fogón, galpones, letrinas o pozos negros, quintas con árboles frutales, cortinas cortavientos de álamos,

\footnotetext{
${ }^{1}$ Sistema que podía ser de tablones labrados a hacha (tipo basas) o palos enteros redondos. En las zonas de unión se estopaban con material vegetal (por ejemplo, musgos) y otros (barro, etcétera).

${ }^{2}$ Conocida también como la técnica del estaqueo, bajareque, embarre o quincha: "Técnica mixta de muros perteneciente a la familia de los entramados, compuesta de una armazón estructural (madera o bambú) rellena con tierra o barro en estado plástico a la cual se le ha añadido fibras vegetales" (Proterra s. f.; cfr. Jorquera 2015).

${ }^{3}$ Sistema de reforzamiento de los ensambles de maderas producto de la escasez o inexistencia de clavos de hierro.

${ }^{4}$ Tapajuntas: cinta o tabla que se coloca sobre la unión de cada pieza de tabla vertical que compone el revestimiento exterior e interior de la casa. También hay casas que presentan ese sistema en el techo.
} 
cercos de palo a pique, corrales, etc., son algunos de los habituales signos remanentes de quienes decidieron establecerse y hacer sus vidas en la zona.

Conociendo la importancia que esos elementos tangibles tienen como reflejo de identidad local del territorio aysenino, los estudios asociados con la arquitectura son escasos y sólo un número acotado de publicaciones (Olivares 2006; Castillo 2015a; Pérez y Castillo 2016; Pérez et al. 2018) han comenzado a visibilizar el valor de ese patrimonio construido, próximo a desparecer. Por ello, es importante seguir reforzando la información existente con el registro de la arquitectura que muestra el proceso de ocupación y apropiación del territorio por medio de la caracterización de la técnica constructiva, de la identificación del volumen propiamente tal, de la definición de los espacios y paisajes circundantes, de los materiales estructurales, de los revestimientos interiores y exteriores, y del uso de herramientas de análisis complementarias, como la dendrocronología (Puchi et al. 2017: 110) para una datación más exacta.

Esos estudios deben abrir nuevas posibilidades para la valoración y protección de lo que se entiende por patrimonio arquitectónico vernáculo en la región de Aysén, tan decaído, desvalorizado e invisibilizado actualmente. Sin lugar a dudas, la investigación interdisciplinaria refuerza la importancia para el territorio de esos elementos construidos, su relevancia como piezas de valor histórico y su condición de huella material como forma particular de habitar.

En contexto, el presente trabajo tiene como objeto central caracterizar los complejos constructivos en madera ubicados en sectores rurales y reforzar el conocimiento y la información existentes sobre la arquitectura vernácula en la región de Aysén, destacar su trascendencia como elemento identitario cultural y difundirla para concienciar a la población local sobre su singularidad y los atributos histórico-culturales que representa ese patrimonio constructivo.

\section{Metodología}

\section{Caracterización general de la zona de estudio}

La región de Aysén se ubica entre los paralelos $44^{\circ}$ y $49^{\circ}$ latitud sur, posee una superficie total de 10698182.7 ha, distribuida en cuatro provincias: Aysén, con 4522485.5 ha; Coyhaique, con 1278532.6 ha; General Carrera, con 1178119.9 ha, y Capitán Prat, con 3719044.8 ha (Castillo 2015a; 2015b). La población regional se distribuye mayoritariamente en las localidades de Puerto Aysén $(29 \%)$ y Coyhaique $(60 \%)$, que concentran $89 \%$ del total. ${ }^{5}$

\footnotetext{
${ }^{5}$ Censo 2012, Instituto Nacional de Estadísticas (INE, Chile), región de Aysén.
}

Las precipitaciones varían desde $240 \mathrm{~mm}$ al año en Chile Chico a 2500 mm anuales en Puerto Aysén y, en términos bioclimáticos, presenta dos zonas definidas: la oceánico-templada fría y la oceánica-trasandina (Hajek y Di Castri 1975: 190-20; Dirección Meteorológica de Chile 2010: 1-3; Hepp y Stolpe 2014).

La superficie de bosque nativo se distribuye principalmente en las provincias de Aysén, con $60.6 \%$, y Capitán Prat, con $21.1 \%$. Las provincias de General Carrera y Coyhaique representan en conjunto sólo $18.1 \%$ de esa categoría (Castillo 2015: 30). Este recurso forestal está compuesto por variadas especies arbóreas que se distribuyen en distinta proporción geográfica dentro del territorio, destacando, entre las más comúnmente utilizadas en la arquitectura: Nothofagus pumilio (lenga), P. uviferum (ciprés de las Guaitecas), Nothofagus betuloides (coigüe de Magallanes), Podocarpus nubigenus (mañío de hojas punzantes), S. conspicua (mañío de hoja corta), D. winteri (canelo), Nothofagus dombeyi (coigüe común), Nothofagus antarctica (ñirre o ñire), Laureliopsis philippiana (tepa), Weinmannia trichosperma (tineo) y Embothriun coccineum (ciruelillo), entre otras.

\section{Levantamiento de datos}

Durante los años 2016, 2017 y 2018, se seleccionaron 22 complejos constructivos en madera insertos en distintos sectores rurales, lo cual hace un total de 41 construcciones en las que destaca la casa principal, la cocina-fogón, el galpón y la letrina (pozo negro). Éstos se encuentran insertos en sectores rurales, y en ellos se recabó la siguiente información:

a) Identificación de construcciones (casa principal, cocina-fogón, galpón y letrina). Se midieron las construcciones en planta y elevación con la ayuda de una huincha de distancia y métrica; conjuntamente, se realizó un levantamiento fotográfico del exterior e interior de cada una de éstas con una cámara Canon EOS D60 y trípode Manfrotto. Se caracterizaron su materialidad constructiva y las dimensiones de las piezas trabajadas (vigas, pies derechos, basas del piso, tablas del revestimiento, encintado, entre otras), y su ubicación espacial se georreferenció utilizando un GPS Oregon 550. Para ese trabajo en particular sólo se da a conocer en detalle un complejo constructivo ubicado en el Fundo San Lorenzo, Lago Cástor, comuna de Coyhaique.

b) Selección e identificación de árboles frutales y álamos para su estudio dendrocronológico. Se determinaron datos básicos de la altura total del árbol y el diámetro del fuste principal; no se utilizaron cronologías de ancho de anillos y sólo se llevó a cabo la estimación de la edad aproximada de 


\begin{tabular}{|c|c|c|c|c|c|}
\hline \multirow{2}{*}{$\begin{array}{l}\text { COMPLEJO CONSTRUCTIVO RURAL } \\
\text { (SECTOR Y COMUNA) }\end{array}$} & \multirow{2}{*}{$\begin{array}{l}\text { NÚM. } \\
\text { CONSTRUCCIONES }\end{array}$} & \multicolumn{3}{|c|}{$\begin{array}{c}\text { UBICACIÓN GEOGRÁFICA } \\
\text { (UTM, WSG } 84 \text { HEMISFERIO SUR) }\end{array}$} & \multirow{2}{*}{$\begin{array}{l}\text { PROPIETARIOS } \\
\text { ORIGINALES }\end{array}$} \\
\hline & & UTM X & UTMY & HuSO & \\
\hline El Claro, Coyhaique & 1 & 726300 & 4946256 & 18 & Rufino Vallejos \\
\hline Sector Reserva, Coyhaique & 3 & 266594 & 4952223 & 19 & s.i.* \\
\hline El Salto, Coyhaique & 3 & 729030 & 4936576 & 18 & Dante Medina \\
\hline Callejón Fernández-Balmaceda, Coyhaique & 2 & 286173 & 4908853 & 19 & Castillo Calderón \\
\hline Lago Cástor, Coyhaique & 2 & 282370 & 4950412 & 19 & Castillo Castillo \\
\hline Lago Frío, Coyhaique & 1 & 277794 & 4945873 & 19 & Natanael Fuenzalida \\
\hline El Claro, Río Ibáñez & 4 & 725126 & 4870973 & 18 & Juan Ojeda \\
\hline Lago La Paren, Río Ibáñez & 1 & 703820 & 4878409 & 18 & Cipriano Alarcón \\
\hline Cerro La Virgen, Coyhaique & 2 & 732053 & 4926882 & 18 & Bernabé \\
\hline El Gato, Coyhaique & 3 & 275712 & 5002582 & 19 & Aros Vega \\
\hline Richard 2-Ñirehuao, Coyhaique & 3 & 284271 & 4981171 & 19 & Vargas Veliz \\
\hline Seis Lagunas, Coyhaique & 1 & 719127 & 4938434 & 18 & Ovando Pérez \\
\hline Cerro Galera, Coyhaique & 3 & 282034 & 4927854 & 19 & s.i.* \\
\hline Lago Thompson, Coyhaique & 1 & 282697 & 4941229 & 19 & Vásquez Hueras \\
\hline El Fraile, Coyhaique & 1 & 732674 & 4936917 & 18 & Fuenzalida Urrutia \\
\hline El Peludo, Coyhaique & 1 & 269394 & 4931082 & 19 & Rivas \\
\hline Lago Largo, Coyhaique & 3 & 276587 & 4989215 & 19 & Millaqueo \\
\hline Mallín Grande, Chile Chico & 1 & 693014 & 4821083 & 18 & Burgos Hermosilla \\
\hline Embarcadero antiguo Mallín Grande, Chile Chico & 1 & 695588 & 4826538 & 18 & Basualto \\
\hline Cerro Negro, Coyhaique & 1 & 274827 & 4945055 & 19 & s.i. $*$ \\
\hline Sector Mano Negra-Villa Ortega, Coyhaique & 2 & 268693 & 4969042 & 19 & Familia Troncoso \\
\hline Lago Monreal, Coyhaique & 1 & 270987 & 4917247 & 19 & Vásquez Oporto \\
\hline Total construcciones & 41 & & & & * Sin información \\
\hline
\end{tabular}

FIGURA 1. Ubicación geográfica de complejos constructivos seleccionados (n: 22). Región de Aysén, Chile (Tabla: Carlos Castillo Levicoy, 2018).

establecimiento. Las mediciones de la altura total del árbol y del diámetro del fuste se hicieron con la ayuda de un hipsómetro Suntto, y con una forcípula y huincha diamétrica, respectivamente.

Se extrajo una muestra de tarugo tomada a $30 \mathrm{~cm}$ de altura desde la base del árbol con un taladro de incremento Finnforce de 12" en $5 \mathrm{~mm}$. Esto se realizó en árboles introducidos y plantados por el primer propietario en torno del conjunto construido, como herramienta de trabajo para obtener la data del asentamiento en el lugar. Posteriormente, las muestras se montaron en molduras de madera y se lijaron, progresivamente, con distintas granulometrías (secuencia de granos grueso a fino), hasta que los anillos de crecimiento se hicieron claramente distinguibles. Con el uso de una lupa estereoscópica, se contó y estimó la edad, y para aquellas muestras en que no se obtuvo el centro de la médula se estimaron los anillos faltantes utilizando la fórmula descrita por Duncan 1989.

c) Entrevistas orales. Se llevaron a cabo entrevistas semiestructuradas a los dueños originales y familiares directos que utilizaron esas construcciones, con una grabadora digital de sonidos PCM D50 Sony, información complementaria que permitió conocer el periodo de construcción de los inmuebles para su posterior contraste con los datos de edad obtenidos de los árboles muestreados. Conjuntamente, dicha información permitió entender el uso y la vida familiar dado en torno de los complejos rurales y la utilización del recurso maderero proveniente del bosque.

\section{Análisis de la información}

Para la ubicación y distribución geográfica de los complejos constructivos rurales, se elaboró una tabla (Figura 1).

Se utilizó el software SigmaPlot $10.0^{\circledR}$ para los gráficos de regresión lineal simple de altura vs. diámetro y diámetro vs. edad estimada de los árboles frutales y de los álamos muestreados (Figuras 2 y 3 ).

Con el software AutoCAD ${ }^{\circledR}$ se llevó a cabo la reconstrucción de la planimetría de dos obras que forman parte del complejo constructivo rural ubicado en el Fundo San Lorenzo, Lago Cástor, comuna de Coyhaique, como caso específico de estudio. El dibujo planimétrico representa el estado de la casa en la década de 


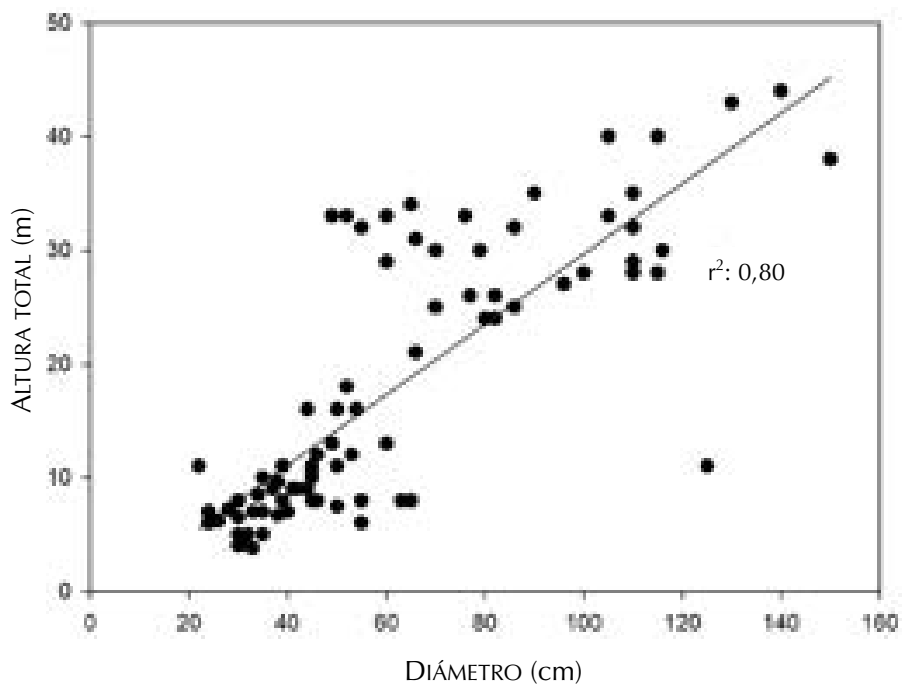

FIGURA 2. Análisis de regresión lineal simple entre el diámetro (basal) y la altura total de los árboles muestreados ( $n$ : 84) (Gráfico: Carlos Castillo Levicoy, 2018).

1930, década de 1940 y principios de la década de 1950 (Figuras 4 y 5 ).

Se elaboraron dos tablas que dan cuenta de las características generales de los complejos rurales ( $n: 22)$ y sus respectivas construcciones en torno de: superficie en metros cuadrados, especies arbóreas a las cuales pertenecen las maderas utilizadas, tipo de cubierta y volumetría, y estado actual (en uso, abandonada, en desmoronamiento, otros). Conjuntamente, se hizo una comparación entre la edad estimada de los árboles y los datos del periodo de construcción dados a conocer en la entrevista oral.

\section{Resultados}

\section{Establecimiento de complejos constructivos}

Se obtuvo una regresión lineal significativa entre la altura total y el diámetro del fuste de los árboles en estudio $\left(r^{2}: 0.80\right.$; Figura 2). Mientras que las edades estimadas tanto para los árboles frutales como para los álamos estudiados van desde los 27 hasta los 91 años, respectivamente (Figura 3).

El establecimiento y construcción más temprano registrado corresponde a los sectores de El Fraile (1926 y 1932), Lago Largo (1930 y 1932), Cerro La Virgen (1932 y 1933), Lago Cástor (1935), El Salto (1935) y Seis Lagunas (1936) (Figura 6). Sólo en el complejo ubicado en el sector El Claro, comuna de Coyhaique, no se pudo constatar el dato de las muestras de tarugo con el entregado en la oralidad, debido principalmente a factores de dureza de la madera para el caso de los árboles de Arce (Acer $s p$.$) , que impidió llegar a la médula (centro del árbol) así$ como a la presencia de pudrición interna avanzada que

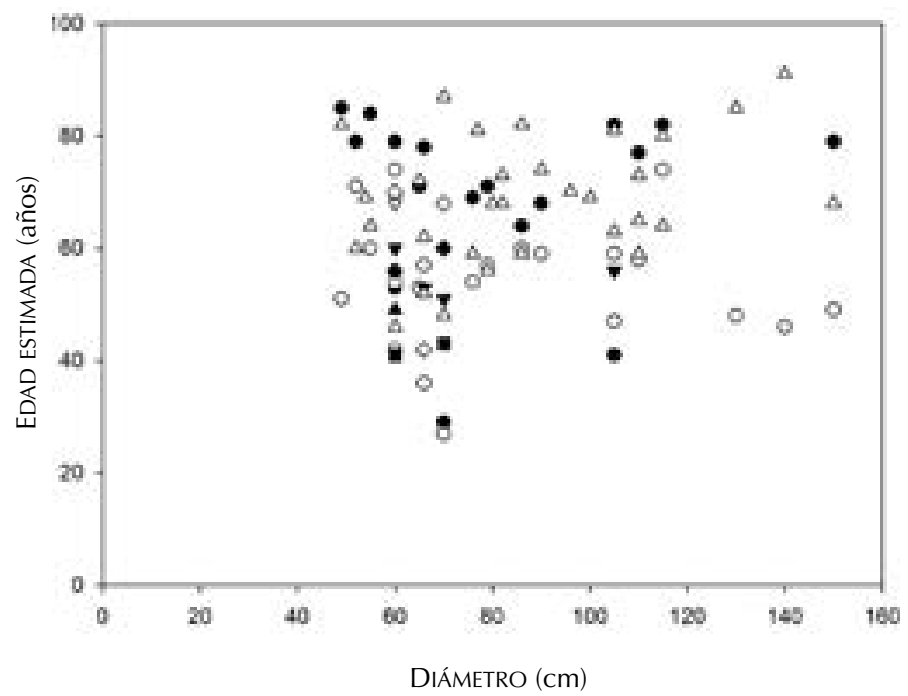

$\begin{array}{llllll}- & \text { Manzano } & \boldsymbol{\square} & \text { Abedul } & \boldsymbol{\Delta} & \text { Pero } \\ \bigcirc & \text { Cerezo } & \square & \text { Abeto } & \nabla & \text { Sauce } \\ \boldsymbol{\nabla} & \text { Ciruelo } & \nabla & \text { Sauco } & & \\ \triangle & \text { Álamo } & \diamond & \text { Arce } & & \end{array}$

FIGURA 3. Análisis de regresión lineal simple entre el diámetro (basal) y la edad estimada de los árboles muestreados $(n, 84)$ (Gráfico: Carlos Castillo Levicoy, 2018).

presentaron los álamos en ese sector. Las demás muestras no mostraron inconvenientes y los datos obtenidos coincidieron con el periodo de construcción dados a conocer a través de la entrevista oral (Figura 7).

Tipos de revestimientos y volumetrías

Los revestimientos típicos encontrados, que se asocian a determinada morfología constructiva, corresponden principalmente al uso de la tejuela artesanal, la tabla vertical con cinta tapajunta, la tabla horizontal traslapada (tingladillo), el machihembrado, el tablón labradoamordazado y estopado, y el adobe. De éstos destaca la tejuela artesanal como uno de los sistemas de revestimiento más comunes encontrados, y se utilizó para revestir tanto el tinglado como el techo de casas, galpones, cocinas-fogón y letrinas o pozos negros, y se combina con los tipos de revestimientos nombrados anteriormente. El tablón labrado y amordazado-estopado como tinglado consiste en una pieza partida, labrada y cuadrada, que se monta una sobre otra y se une en sus vértices con cortes; para reforzar su unión, pueden utilizarse clavos de madera como sistema de anclaje. Esa forma de tinglado rústico se caracteriza por ser de los primeros sistemas constructivos que se realizaron en la región; actualmente quedan muy pocos vestigios, que sólo se observan en contados sectores rurales (campos).

Los tipos de cubierta de "un agua, dos aguas y cuatro aguas", la volumetría simple "rectangular" y compuesta 


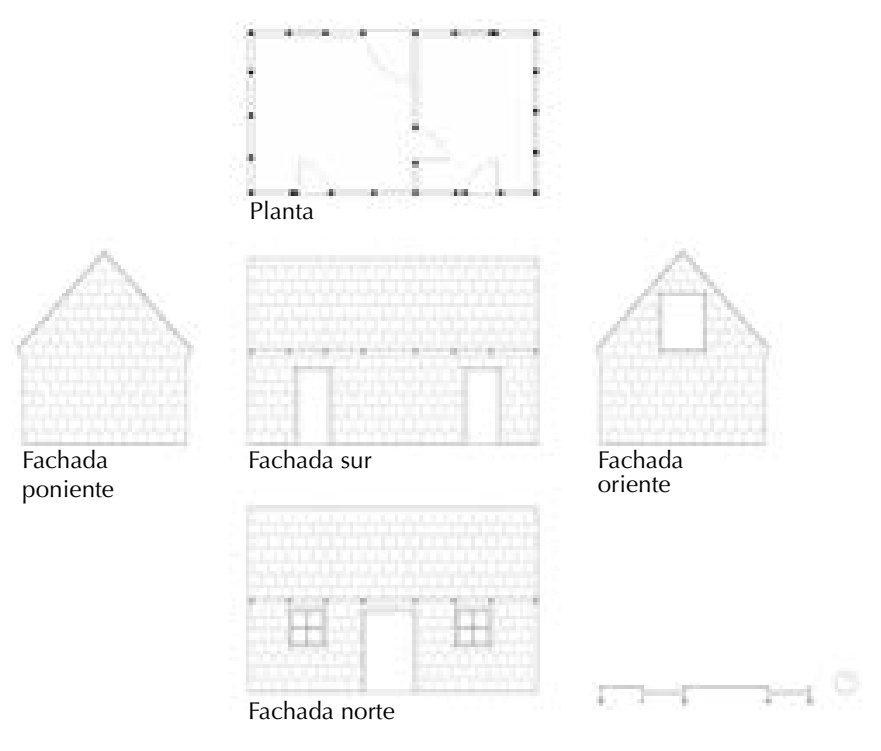

FIGURA 4. Planimetría primera casa habitación de la familia Castillo Castillo, Fundo San Lorenzo, Sector Lago Cástor, comuna de Coyhaique, región de Aysén, Chile (Gráfico: Constanza Pérez Lira, 2018).

en forma de $L$ y $U$, fueron las que más comúnmente se observan dentro de los complejos estudiados (Figura 6). La autoconstrucción domina en todos los complejos rurales y no existen dibujos a mano alzada ni planos que ayuden a ver y determinar algún tipo de diseño planificado.

\section{Caracterización general de la madera (dimensiones)}

Las construcciones más antiguas estudiadas en los distintos complejos presentan piezas estructurales labradas a hacha de distinto espesor, ancho y largo, mientras que las más recientes poseen piezas aserradas en mayor proporción. Esto da cuenta, asimismo, de la distinta disponibilidad al acceso de tecnología y recursos económicos para trabajar la madera que poseían los pobladores en los distintos sectores estudiados.

Las vigas labradas van de los $3 \times 3$ a $4 \times 8$ (las medidas siguientes, salvo que se indique lo contrario, van en pulgadas); los pies derechos labrados, de $3 \times 3$ a 8 $\times 8$; las piezas labradas de la cercha, de $3 \times 4$ a $4 \times$ 5 . Las tablas de traslapo horizontal (tingladillo), desde $4 \times 2$ hasta $20 \times 3$; las tablas de traslapo vertical, de 4 $\times 2$ a $8 \times 1$, con cintas tapajuntas de $1 \times 1$ a $3 \times 2$; el machihembrado, de $3 \times 1$. Cintas de entejuelado, ${ }^{6}$ de 3 $\times 1$ a $3 \times 2$, y espacias a cada $20 \mathrm{~cm}$. También destaca la presencia de tablones tanto labrados cumpliendo una función de divisores de muros interiores de $14 \times 3$, como amordazados y estopados a manera de tinglado horizontal de $7 \times 11$, aproximadamente. Los pollos y zoquetes de madera, elementos característicos de la casa principal de los complejos (sistema de piso ventilado), tienen una

\footnotetext{
${ }^{6}$ El entejuelado es el proceso de colocación de la tejuela artesanal en el tinglado (pared) o techo de la construcción.
}

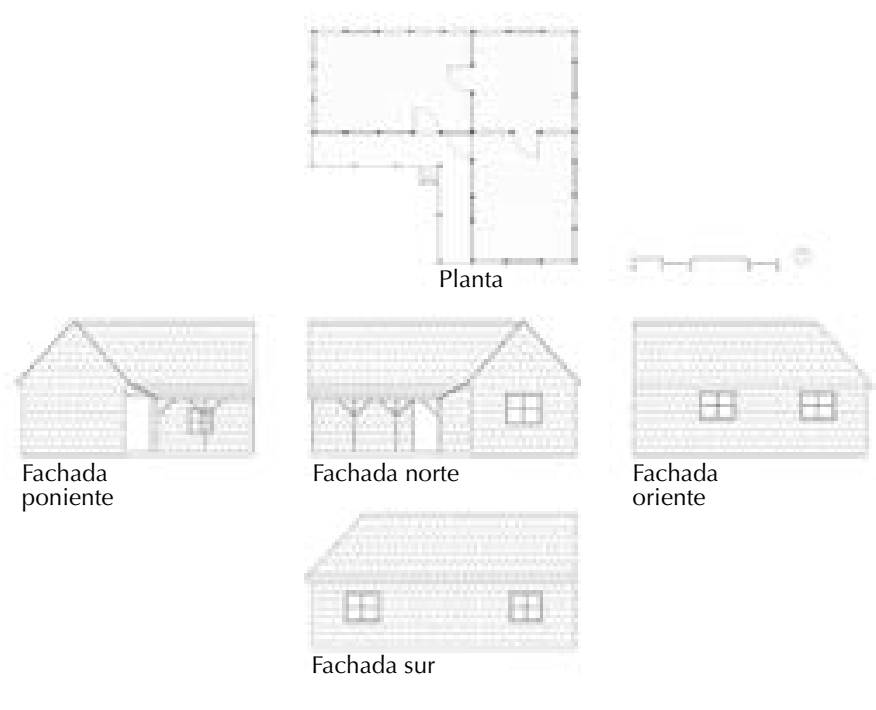

FIGURA 5. Planimetría segunda casa habitación de la familia Castillo Castillo, Fundo San Lorenzo, Sector Lago Cástor, comuna de Coyhaique, región de Aysén, Chile (Gráfico: Constanza Pérez Lira, 2018).

altura, que sobresale del suelo, de 25 a $30 \mathrm{~cm}$, y diámetro variable desde 15 hasta $25 \mathrm{~cm}$, respectivamente. Por otro lado, cabe destacar la presencia de palos partidos y apique en los cierres perimetrales del emplazamiento de quintas y construcciones.

Dentro de las especies arbóreas mayormente utilizadas en esos complejos, destacan: lenga (N. pumilio), coigües ( $N$. betuloides y N. dombeyi), ñirre (N. antarctica) y mañio (S. conspicua), mientras que la presencia de madera de ciprés de las Guaitecas (P. uviefrum) es menor (Figura 7).

\section{Complejo constructivo del Fundo San Lorenzo, Lago Cástor}

El complejo constructivo ubicado en el fundo San Lorenzo, sector de Lago Cástor, se caracteriza por la existencia de dos casas de madera, construidas a principios de la década de 1930 (Figura 4) y finales de la década siguiente (Figura 5). Ambas poseen una cubierta de dos aguas, la primera, una de volumetría simple de planta rectangular; y la segunda, una especial por su volumetría compuesta de planta en forma de $L$ con corredor. Pertenecieron a los pobladores Lorenzo Castillo y Sara Fuenzalida (ambos fallecidos), quienes llegaron al sector a finales de la década de 1910, aproximadamente, provenientes de la zona centro-norte de Chile, pasando por territorio argentino e ingresando en la región de Aysén por Aldea Beleiro, provincia del Chubut-Argentina paso fronterizo El Triana. Ambas casas, con superficies de 27 y $59 \mathrm{~m}^{2}$, tienen la peculiaridad de estar construidas con madera de lenga (N. pumilio) $y$, en menor proporción, ñirre ( $N$. antartica); respecto de su materialidad, destaca el uso de la tejuela artesanal de lenga como revestimiento de techos y tinglados, mientras 


\begin{tabular}{|c|c|c|c|c|c|}
\hline $\begin{array}{c}\text { TIPO DE } \\
\text { CONSTRUCCIÓN }\end{array}$ & N & $\begin{array}{l}\text { SUPERFICIE PROMEDIO } \\
\pm \mathrm{SD}\left(\mathrm{M}^{2}\right)\end{array}$ & ESPECIE ARBÓREA UTILIZADA & $\begin{array}{c}\text { ESTADO ACTUAL } \\
\text { DE LAS CONSTRUCCIONES }\end{array}$ & $\begin{array}{l}\text { TIPO DE CUBIERTA } \\
\text { Y VOLUMETRÍA }\end{array}$ \\
\hline Casa principal & 24 & $55 \pm 38$ & $\begin{array}{l}\text { N. pumilio, N. antartica, P. uviferum, } \\
\text { S. conspicua y N. dombeyi }\end{array}$ & \multirow{4}{*}{$\begin{array}{l}\text { Casas: Las casas que están en } \\
\text { desuso se encuentran en un } \\
\text { avanzado estado de deterioro } \\
\text { de la madera de revestimiento y } \\
\text { estructural. Algunas son utilizadas } \\
\text { actualmente como bodega para } \\
\text { guardar forraje y otros enseres } \\
\text { por parte de los dueños actuales. } \\
\text { Las que están habitadas, el } \\
\text { revestimiento del techo presenta } \\
\text { deterioro y esto ha llevado a } \\
\text { colocar planchas de zinc sobre } \\
\text { la tejuela, mientras que la madera } \\
\text { estructural y de revestimiento en } \\
\text { el tinglado (exterior e interior) } \\
\text { presentan un menor deterioro; } \\
\text { Cocina-fogón: todas se encuentran } \\
\text { en desuso, con un marcado } \\
\text { avance de deterioro tanto de la } \\
\text { madera de revestimiento de techo } \\
\text { y tinglado, y estructural; galpones } \\
\text { y letrinas: comparten la misma } \\
\text { situación de desuso y deterioro } \\
\text { de su madera de revestimiento y } \\
\text { estructural. }\end{array}$} & $\begin{array}{l}\text { Dos y cuatro aguas, } \\
\text { simple y compuesta } \\
\text { en " } U^{\prime \prime} y^{\prime \prime}{ }^{\prime}\end{array}$ \\
\hline Cocina fogón & 10 & $21 \pm 7$ & $\begin{array}{l}\text { N. pumilio, N. antarctica, N. dombeyi } \\
\text { y N. betuloides }\end{array}$ & & $\begin{array}{l}\text { Una y dos aguas, } \\
\text { simple }\end{array}$ \\
\hline Galpón & 4 & $137 \pm 75$ & N. pumilio, N. antarctica y N. dombeyi & & Dos aguas y simple \\
\hline $\begin{array}{l}\text { Pozo negro y/o } \\
\text { letrina }\end{array}$ & 3 & $4 \pm 2$ & $\begin{array}{l}\text { N. pumilio, N. antarctica, N. dombeyi } \\
\text { y N. betuloides }\end{array}$ & & $\begin{array}{l}\text { Una y dos aguas, } \\
\text { simple }\end{array}$ \\
\hline Total & 41 & & & & \\
\hline
\end{tabular}

FIGURA 6. Caracterización general de construcciones estudiadas (n: 41), región de Aysén, Chile (Tabla: Carlos Castillo Levicoy, 2018).

que la madera estructural es labrada a hacha y la madera interior (piso, cielo y paredes), aserrada. Las vigas son de 6 $\times 5$ (medidas, en pulgadas, salvo indicación en contrario) y pies derechos de $4 \times 4$ para la casa construida en 1930 y con vigas labradas de $5 \times 4$ y pies derechos labrados de $5 \times 4$ para la casa de finales de 1940. Ambas construcciones poseen cintas (entejuelado techo y tinglado) de $3 \times$ 2 , separadas cada $20 \mathrm{~cm}$, y el piso, tablas de $10 \times 2$, con basas labradas de $6 \times 5$, montado sobre pollos de madera (piso ventilado). La madera de revestimiento y estructural de éstas se encuentra en avanzado estado de deterioro, y actualmente los dueños las utilizan como bodega para guardar forraje y otros enseres.

Los siguientes testimonios dan cuenta de una parte de la vida familiar ligada a ese complejo:

En Lago Cástor, el finado Lorenzo junto a la abuela Sara nos pasaron una mediagüita (Figura 4), llevamos unas pilchitas, unas cosas que me dio mi mamá, de a poquito nos afirmamos junto a mi marido Crispín Castillo Fuenzalida (q. e. p. d.). En esos años no habían muchas casas, puras ranchitas en el sector. Con los años hicimos una buena casa (Figura 5), un fogón, el galpón, todos con tejuelas que yo misma hice con ayuda de mi papá, Rómulo Castillo Otárola (q. e. p. d.) y con mi hijo Crispín Segundo Castillo Castillo. La mediagua tenía dos piezas, la casa nueva tenía un corredor, una pieza grande, un comedor y la cocina. En el campo hacía quesos y mis hijos los más grandes ayudaban a ordeñar las vacas el día sábado y domingo, ya que en la semana estaban en la escuela de Lago Pólux. Se vendía harto queso que salía de la ordeña de ocho vacas, cuatro del finado Santiago y las otras cuatro eran de nosotros. Hacíamos mantequilla. Teníamos una huerta grande, donde había grosellas, mosquetas, parras (zarzaparrilla), ruibarbos; sembrábamos ajos, cebollas, papas, se daba de todo [Elica Castillo Cárdenas, 84 años de edad, comunicación personal, Coyhaique, octubre de 2017].

La casa vieja que perteneció a mis abuelos Lorenzo y Sara fue autoconstrucción como la más nueva que hicimos con mi mamá. No hubo dibujos, ni planos. Ambas casas tienen los pies derechos, las vigas y piezas de las cerchas labradas a hacha, el resto de madera como tablas, las cintas, etc., son aserradas. No todos los pobladores tenían acceso a los aserraderos para trabajar sus maderas y muchos aserraron con la sierra a brazo y así construían. Se hacían las bazas labradas a hacha y luego éstas se marcaban y aserraban. Había buenos trabajadores de la madera en esos años. Los abuelos, cuando llegaron al sector vivieron en un rancho de canogas y palos partidos, con piso de tierra y, a lo más, tablones labrados. Gran parte de las familias Ilegadas al sector y alrededores construyeron así provisoriamente mientras se establecían y construían una casa con mejores comodidades [Crispín Castillo Castillo, 64 años de edad, comunicación personal, Coyhaique, noviembre de 2017].

El primer rancho más característico que construyeron los pobladores llegados a la zona fue el de canogas y palo 


\begin{tabular}{|c|c|c|c|c|c|c|}
\hline $\begin{array}{l}\text { COMPLEJO CONSTRUCTIVO } \\
\text { RURAL (SECTOR Y COMUNA) }\end{array}$ & $\begin{array}{l}\text { NOMBRE COMÚN } \\
\text { ÁRBOL }\end{array}$ & N & $\begin{array}{l}\text { ALTURA TOTAL } \\
(\mathrm{M})\end{array}$ & $\begin{array}{l}\text { DIÁMETRO } \\
(\mathrm{CM})^{\prime \prime}\end{array}$ & $\begin{array}{l}\text { EDAD ESTIMADA } \\
\text { (AÑOS) }\end{array}$ & $\begin{array}{l}\text { PERIODO CONSTRUCCIÓN } \\
\text { SEGÚN ORALIDAD** }\end{array}$ \\
\hline \multirow{3}{*}{ El Claro, Coyhaique } & Arce & 2 & 31 & 113 & 1947,1949 & \multirow{3}{*}{ Década de 1920} \\
\hline & Cerezo & 1 & 13 & 60 & 1964 & \\
\hline & Álamo & 1 & 38 & 150 & 1949 & \\
\hline \multirow{3}{*}{ Sector Reserva, Coyhaique } & Álamo & 8 & 32 & 81.5 & $\begin{array}{l}\text { 1948*, 1953*, 1954*, 1958*, } \\
\text { 1961, 1965, 1969, 1971 }\end{array}$ & \multirow{3}{*}{ Décadas de 1940 y 1950} \\
\hline & Manzano & 2 & 6.7 & 31.5 & $1958 *, 1961$ & \\
\hline & Peral & 1 & 6.7 & 38 & 1968 & \\
\hline \multirow{3}{*}{ El Salto, Coyhaique } & Cerezo & 3 & 8 & 40 & 1958,1960 & \multirow{3}{*}{ De 1935 a 1940} \\
\hline & Manzano & 1 & 7.5 & 50 & 1935*, 1939* & \\
\hline & Ciruelo & 1 & 6 & 24 & 1957 & \\
\hline \multirow{2}{*}{$\begin{array}{l}\text { Callejón Fernández- } \\
\text { Balmaceda, Coyhaique }\end{array}$} & Álamo & 2 & 33.5 & 62.5 & $1947^{*}, 1947^{*}$ & \multirow{2}{*}{ Década de 1940} \\
\hline & Manzano & 2 & 6.7 & 27 & $1938^{*}, 1940^{*}$ & \\
\hline Lago Cástor, Coyhaique & Álamo & 3 & 32.6 & 52 & 1935*, 1953*, 1957* & Década de 1930 y 1950 \\
\hline \multirow{5}{*}{ El Claro, Río Ibáñez } & Cerezo & 2 & 9.5 & 34.5 & 1975,1990 & \multirow{5}{*}{ Década de 1960} \\
\hline & Manzano & 2 & 9 & 23 & 1964*, 1988 & \\
\hline & Abedul & 1 & 16 & 35 & 1976 & \\
\hline & Abeto & 1 & 18 & 52 & $1963^{*}$ & \\
\hline & Sauco & 2 & 7 & 29.5 & 1957*, 1961*, 1968* & \\
\hline \multirow{3}{*}{ Lago La Paren, Río Ibáñez } & Manzano & 1 & 16 & 44 & $1946^{*}$ & \multirow{3}{*}{ Década de 1930} \\
\hline & Cerezo & 3 & 10.6 & 46 & $1943 *, 1957,1959,1960,1963$ & \\
\hline & Ciruelo & 2 & 8.5 & 45 & 1952 & \\
\hline \multirow{3}{*}{$\begin{array}{l}\text { Cerro La Virgen, } \\
\text { Coyhaique }\end{array}$} & Manzano & 3 & 11 & 46 & 1932*, 1933*, 1938* & \multirow{3}{*}{ Década de 1950} \\
\hline & Cerezo & 1 & 6 & 55 & 1946* & \\
\hline & Abedul & 1 & 16 & 50 & 1974 & \\
\hline \multirow{2}{*}{ Seis Lagunas, Coyhaique } & Manzano & 2 & 8 & 54 & $1938^{*}, 1946$ & \multirow{2}{*}{ Década de 1930} \\
\hline & Álamo & 3 & 38 & 103 & 1936*, 1937*, 1943 & \\
\hline \multirow{3}{*}{ El Fraile, Coyhaique } & Ciruelo & 3 & 8 & 58.3 & $1961,1964,1966$ & \multirow{3}{*}{$\begin{array}{l}\text { Primeras construcciones } \\
\text { finales década de } 1910 \text { y } \\
\text { principios década de } 1920 \\
\text { (no existen vestigios). Casa } \\
\text { década de } 1940 .\end{array}$} \\
\hline & Cerezo & 6 & 9.2 & 42 & $\begin{array}{l}\text { 1943*, 1957, 1958, 1966, } \\
\text { 1968, 1970 }\end{array}$ & \\
\hline & Álamo & 2 & 33.5 & 135 & 1926*, 1932* & \\
\hline \multirow[b]{3}{*}{ El Peludo, Coyhaique } & Manzano & 2 & 4.5 & 30 & 1956*, 1969 & \multirow{3}{*}{$\begin{array}{l}\text { Primera construcción a } \\
\text { finales de la década de } \\
1940 \text { y principios de la } \\
\text { década de } 1950 . \text { Segunda } \\
\text { casa de mediados de la } \\
\text { década de } 1960 .\end{array}$} \\
\hline & Álamo & 1 & 21 & 66 & 1955* & \\
\hline & Cerezo & 2 & 7 & 32 & 1969,1971 & \\
\hline \multirow{2}{*}{$\begin{array}{l}\text { Lago Largo-Ñirehuao, } \\
\text { Coyhaique }\end{array}$} & Álamo & 7 & 24 & 76 & $\begin{array}{l}\text { 1932*, 1949, 1949, 1930*, } \\
\text { 1935*, 1936*, 1944 }\end{array}$ & \multirow{2}{*}{$\begin{array}{l}\text { Casa abuela década de } \\
1930 \text { en adelante, casa } \\
\text { padres década de } 1940 \\
\text { en adelante. }\end{array}$} \\
\hline & Manzano & 1 & 8 & 46 & $1935^{*}$ & \\
\hline $\begin{array}{l}\text { Embarcadero antiguo Mallín } \\
\text { Grande, Chile Chico }\end{array}$ & Álamo & 5 & 28 & 106 & $\begin{array}{l}\text { 1944*, 1947*, 1948*, 1952*, } \\
\text { 1953* }\end{array}$ & $\begin{array}{l}\text { Casa construida en la } \\
\text { década de } 1950 .\end{array}$ \\
\hline \multirow{5}{*}{ Lago Monreal, Coyhaique } & Sauce & 1 & 11 & 125 & 1949* & \multirow{4}{*}{$\begin{array}{l}\text { Casa original construida } \\
\text { a finales de la década de } \\
\text { 1940. La segunda casa fue } \\
\text { construida aproximadamente } \\
\text { a finales de la década de } \\
1950 .\end{array}$} \\
\hline & Cerezo & 1 & 5 & 35 & 1981 & \\
\hline & Sauco & 1 & 5 & 32 & 1975 & \\
\hline & Arce & 1 & 11 & 45 & 1975 & \\
\hline & Total árboles & 84 & & & & \\
\hline
\end{tabular}

“ Diámetro promedio medido a los $30 \mathrm{~cm}$ de altura y donde se extrajo el tarugo para determinar edad del árbol.

* Árboles cuyo dato de fechado de anillos de crecimiento anual concuerda con los datos del periodo de construcción de la arquitectura vernácula dados a conocer a través de la oralidad.

** Periodo de construcción aproximado dados a conocer a través de la oralidad.

FIGURA 7. Caracterización del arbolado de los complejos estudiados ( $n$ : 14; sólo en estos complejos se encontró la presencia de quinta con árboles frutales y álamos). Región de Aysén, Chile (Tabla: Carlos Castillo Levicoy, 2018). 


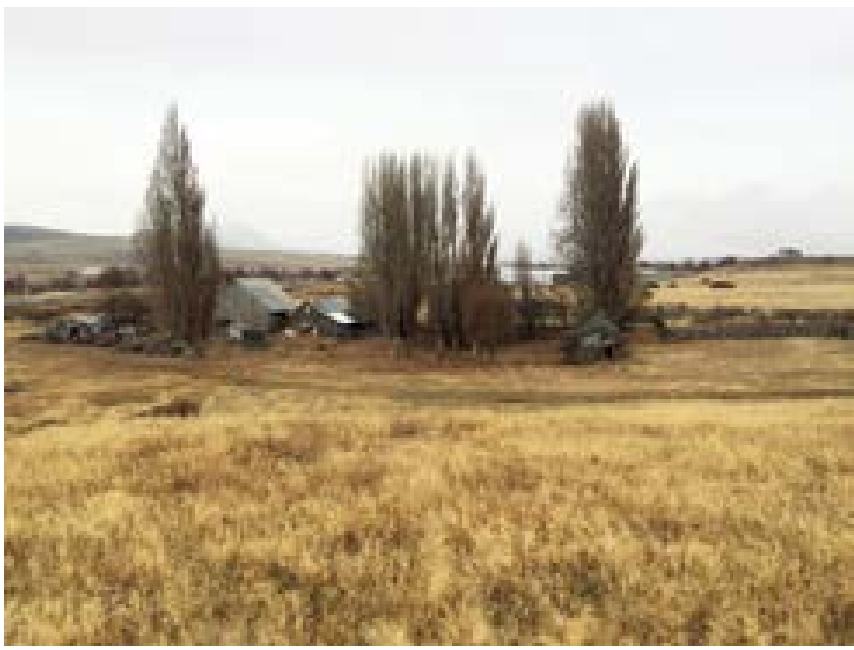

FIGURA 8. Complejo constructivo rural. Sector El Salto, comuna de Coyhaique, región de Aysén, Chile (Fotografía: Carlos Castillo Levicoy, 2017).

partido, piso de tierra, todos construían así. También de palo amordazado, clavados con clavos de madera. Otros con pared francesa y de adobe. Luego ya construyeron con tejuelas y tabla aserrada, primeramente a sierra a brazo y luego ya en aserraderos movidos con molinos a agua. Las tejuelas de muchas construcciones que se hicieron en el sector de Lago Cástor, Pólux y Lago Frío las hizo Rómulo Castillo Otárola (q. e. p. d.) y Atilio Soto (q. e. p. d.), ellos eran los tejueleros que habían en esos años. Se trasladaban de un lugar a otro, haciendo la tejuela, construyendo galpones, muebles, las casas, las cocinas fogón, las letrinas. Hoy todavía quedan construcciones de éstas. Junto a las pocas pilchas que traían los pobladores, Ilegaron los álamos para hacer las cortinas, los frutales para armar las quintas, los yuyos, también algunas semillas para sembrar, ya que muchos hicieron sus huertas. Los cierres de las casas y de la huerta, todo era de palo partido y a pique, para esto los viejos hacían un canal y luego los enterraban bien juntos, era harta pega para hacer estos cierres. También se ayudaban con bueyes. Los cierres del campo (incluidos potreros) con cerco cajón y con alambrados [Elías Urrutia Contreras, 78 años de edad, comunicación personal, El Fraile-Coyhaique, septiembre de 2017].

\section{Análisis y discusión}

Una de las características fundamentales de cada complejo constructivo estudiado (Figura 8) recae en la existencia de distintos espacios que se utilizaban para determinadas actividades. En contexto, se destaca la casa principal (Figura 9) como el recinto en torno del cual giraba la vida familiar; la cocina-fogón (Figura 10), donde se hacían los asados, se conservaba y ahumaba la carne, se maduraban los quesos, se guardaban algunos aperos, entre otros usos; el galpón (Figura 11), necesario para la guarda del forraje, los enseres agrícolas, los aperos, y como protec-

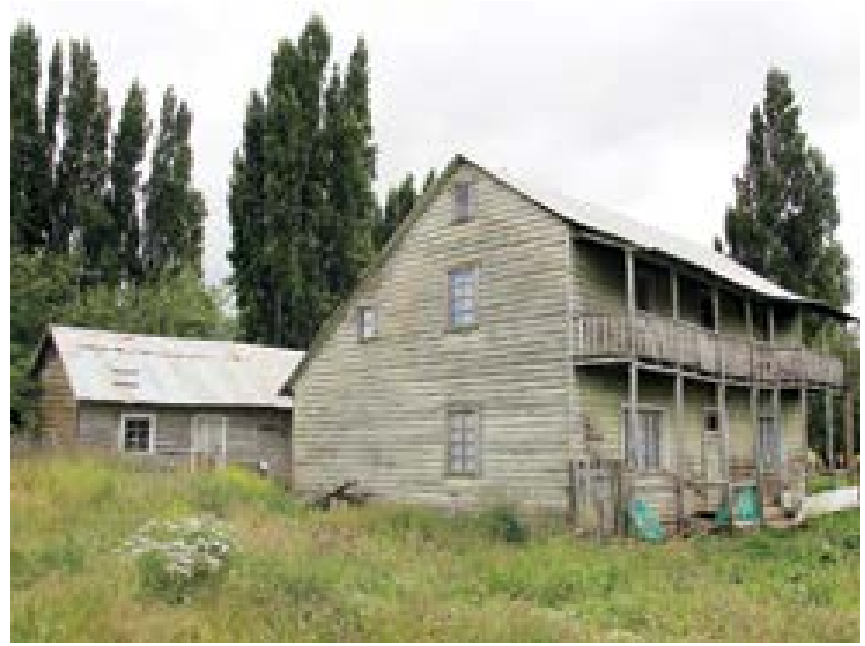

FIGURA 9. Casa habitación principal. El Salto, comuna de Coyhaique, región de Aysén, Chile (Fotografía: Carlos Castillo Levicoy, 2018).

ción del ganado ovino, equino y bovino, cuando las condiciones climáticas lo ameritaban; por otro lado, la letrina o pozo negro (Figura 12), retirada de la casa, cumplía la función de baño; la quinta, compuesta por árboles frutales de manzanos, cerezos, ciruelos, perales, guindos, entre otros, los cuales producían la fruta para la elaboración de mermeladas, consumo en general y para hacer de los manzanos la típica chicha, bebida producida principalmente por las familias Ilegadas desde Chiloé, estaba cercada con palos partidos y apique y, en ocasiones, con tablas o tablones aserrados; el corral tipo "cerco cajón", donde se realizaban diferentes actividades con el ganado (ovino, equino, bovino y caprino), como marcar, capar, señalar, seleccionar y apartar, contar, amansar, y los álamos como cortinas cortavientos, que cumplían un papel principal de protección para las construcciones, la quinta, la huerta y los potreros, entre otros.

Los pobladores que decidieron asentarse de forma permanente en la región de Aysén debieron acostumbrarse a un continuo proceso de transformación y adaptación a las condiciones climáticas y geográficas extremas existentes. Una de las más habituales y concretas que adquirió ese proceso de establecimiento fue la autoconstrucción, donde los pobladores debieron levantar sus propias viviendas y todas aquellas estructuras necesarias para la subsistencia. El medio brindó los recursos necesarios: la madera, y los hombres y mujeres — traídos desde sus lugares de procedencia-, el oficio y las técnicas, aprendidos y transmitidos de generación en generación. Esto dio como resultado una arquitectura autoconstruida de carácter único, que representa en sí misma el gesto de sobrevivencia de aquellos primeros pobladores que decidieron adentrarse en territorio aysenino. Estos inmuebles dieron resguardo, abrigo y funcionalidad durante los primeros años de establecimiento.

Junto con cada poblador que Ilegó al territorio, los árboles frutales y los álamos fueron parte importante de 


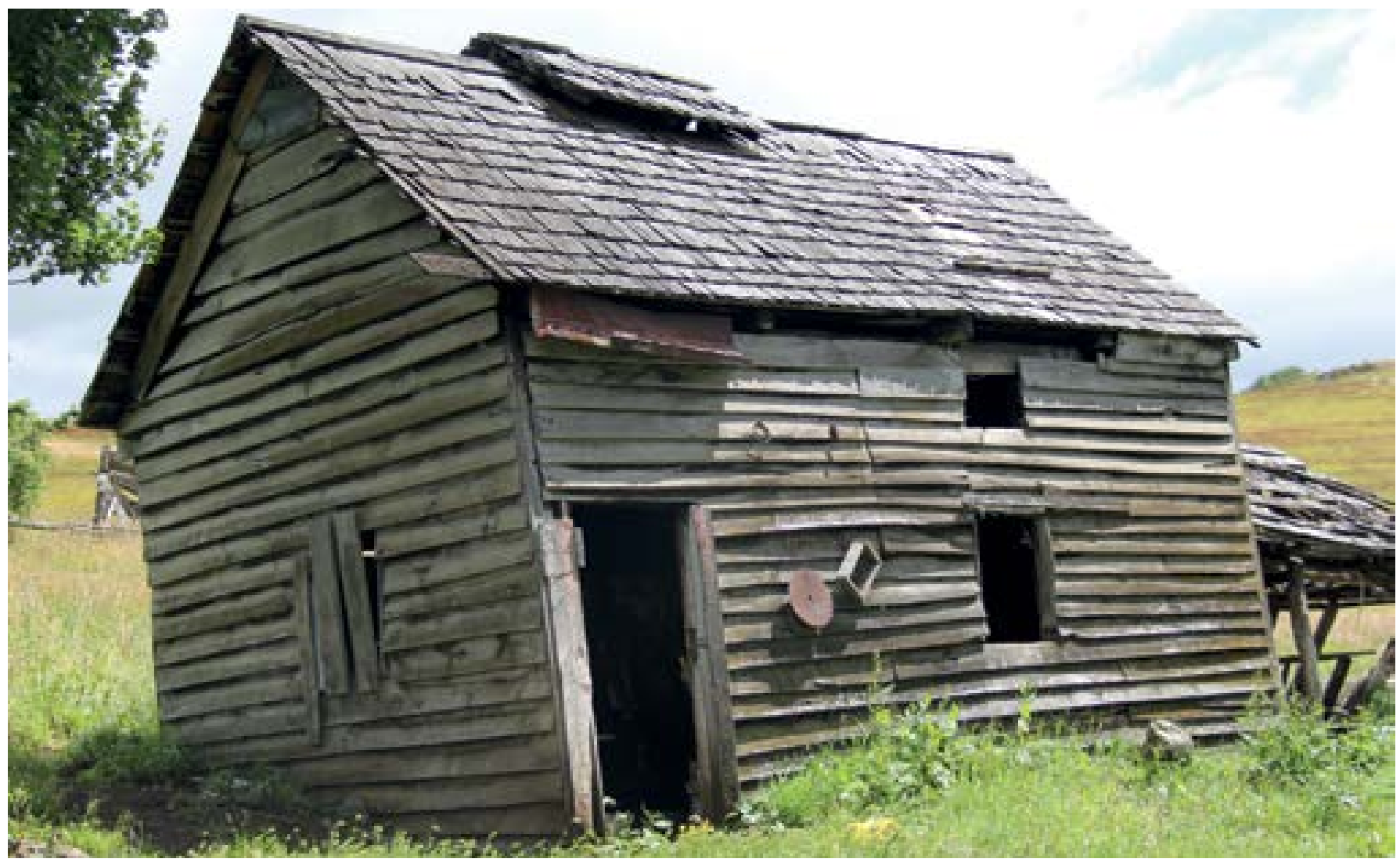

FIGURA 10. Galpón. Lago Largo-Ñirehuao, comuna de Coyhaique, región de Aysén, Chile (Fotografía: Carlos Castillo Levicoy, 2018).

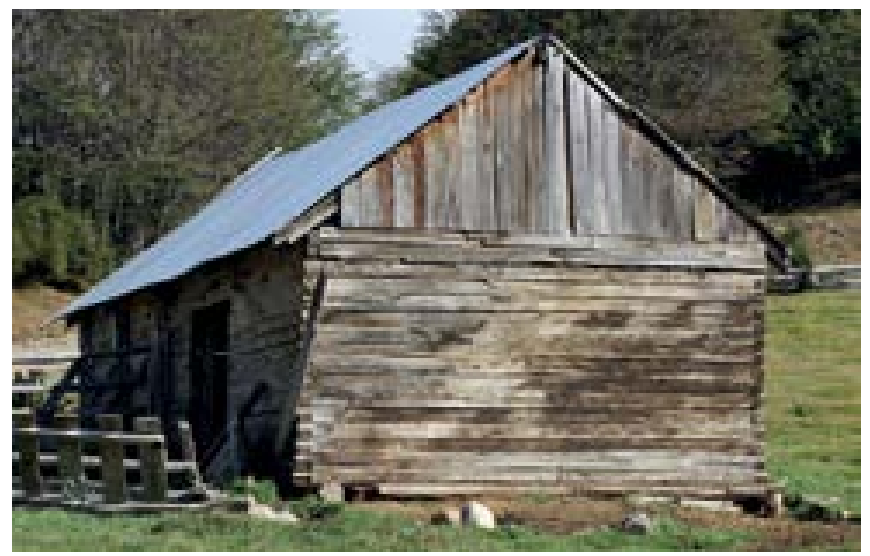

FIGURA 11. Cocina fogón. El Salto, comuna de Coyhaique, región de Aysén, Chile (Fotografía: Carlos Castillo Levicoy, 2017).

los enseres. En cada lugar donde se comenzó a construir, se fue estableciendo la quinta, con manzanos, cerezos y ciruelos, entre otros; conjuntamente, se plantaron los álamos, cuya función dentro del complejo constructivo ya se expuso líneas arriba.

En contexto, la determinación de la edad de esos árboles en el presente estudio permite contar con un registro complementario que abre la oportunidad de precisar y respaldar los datos entregados oralmente, resaltando la importancia de la utilización de distintas fuentes de

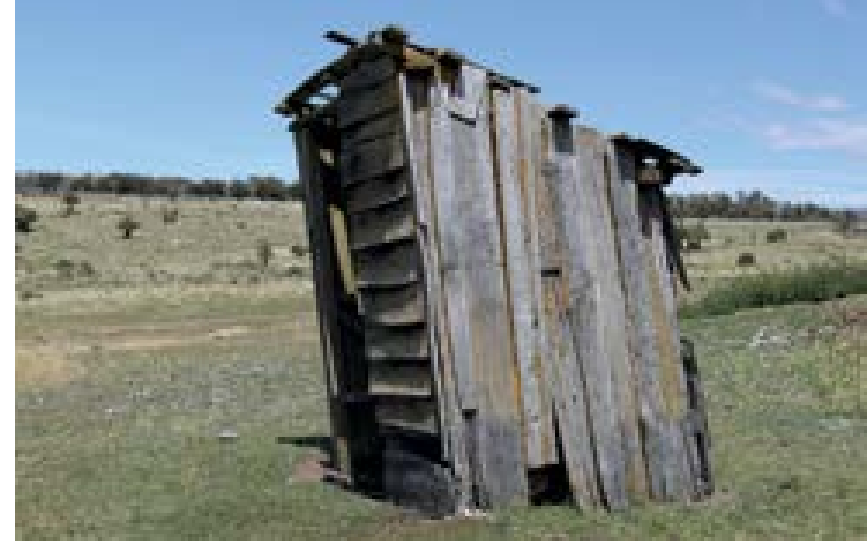

FIGURA 12. Letrina o pozo negro. Interior callejón Fernández, Balmaceda, comuna de Coyhaique, región de Aysén, Chile (Fotografía: Carlos Castillo Levicoy, 2017).

información y análisis disponibles para determinar las fechas exactas o aproximadas de construcción del complejo arquitectónico vernacular estudiado, además de contextualizar de mejor forma la información histórica de ese tipo de estudios (Puchi et al. 2017: 119). Debido a la precariedad o inexistencia del estudio de anillos de crecimiento de los árboles como método de datación complementaria en los estudios de arquitectura vernácula en el territorio aysenino, urge reforzar ese tipo de trabajos en la actualidad para complementar los registros de las 
tipologías constructivas y sus distintas morfologías, junto con el conocimiento local.

Respecto de los sistemas constructivos estudiados, una de las particularidades de los sistemas de revestimiento (exterior e interior) y de la madera estructural aserrada encontrada en los complejos estudiados ha sido su estrecha relación con los sistemas de aserrío. En las primeras décadas del siglo xx destaca el Ilamado con sierra a brazo, en la cual las piezas se obtenían de una basa labrada y cuadrada colocada sobre un borriquete. También se empleó el sistema de aserrío con molino, aprovechando la fuerza hidráulica, y, desde 1937 (aproximadamente) en adelante, con la llegada y el funcionamiento masivo de los motores a vapor, conocidos comúnmente como locomóviles, mejoró el sistema de producción de madera aserrada, de acuerdo con la alta demanda constructiva que había en la región (Castillo 2015a: 15). A esto se suma la llegada de los motores de combustible (de mediados de la década de 1940 en adelante), con lo cual el aprovechamiento maderero mejoró notablemente, así como los acabados de las construcciones, pues se comenzó a utilizar madera de menor dimensión, y la típica construcción tradicional dio paso a construcciones seriadas muy distintas de las de los comienzos de las localidades que existen actualmente en la región de Aysén (Castillo 2015a: 9).

La necesidad de profundizar estudios en el territorio

El desconocimiento y la pérdida de ciertas prácticas tradicionales, en estrecha vinculación con los oficios constructivos adquiridos y traspasados por generaciones, ejecutadas por la gran mayoría de los pobladores que se establecieron en el territorio aysenino, han llevado a que actualmente exista un quiebre entre generaciones respecto de los estilos, las formas constructivas y sus usos. Las construcciones tradicionales en madera, en ocasiones conjugada con otros materiales nobles, como el adobe y el ladrillo cocido, ${ }^{7}$ están desapareciendo del paisaje rural y urbano, por lo que queda un número reducido de ejemplares que se resisten a la demolición causada por las prácticas contemporáneas de habitabilidad y construcción. Esos sistemas constructivos vernáculos posiblemente desaparecerán, como acontece con muchas tecnologías constructivas del saber popular (Batista 2011: 86; Pérez et al. 2017: 31-35); por ello, es importante pesquisar, analizar y dejar registro de ese patrimonio material e inmaterial importantísimo como elemento identitario de un territorio dado. Por eso mismo, existe la creciente necesidad de profundizar los estudios tendentes a comprender cómo se ha gestado la ocupación del territorio aysenino, en conjunto con el conocimiento histórico de las técnicas de au-

\footnotetext{
${ }^{7}$ Conjugación de materiales dados principalmente en zonas orientales
} de la región (límite con territorio argentino). toconstrucción dominadas por sus pobladores. Ese trabajo permitirá, sin lugar a dudas, integrar y contextualizar de mejor forma la escasa información existente y dará cuenta de la ocupación y utilización de los recursos disponibles (por ejemplo, la madera) y del modo en que se ha generado una transformación del paisaje habitado en la región.

La trayectoria constructiva en el territorio aysenino (la casa refugio, la casa definitiva y la casa vieja) alude a las expectativas de habitabilidad de los pobladores, una entidad dinámica cuya dimensión material y simbólica está sujeta a transformaciones constantes, en consonancia con los cambios en las necesidades y deseos de sus ocupantes, y, por último, hace referencia al movimiento permanente de decaimiento y de arruinamiento que afecta indistintamente a la arquitectura como materia (Pérez et al. 2017; Edensor 2016; Batista 2011).

\section{Agradecimientos}

A las familias propietarias de los complejos estudiados de las comunas de Coyhaique (Lago Cástor, Lago Frío, Cerro La Virgen, El Claro, El Salto, Callejón FernándezBalmaceda, Richard 2, Lago Largo y el Gato); de Río Ibáñez (Lago La Paren y el Claro-lbáñez), y de Chile Chico (Mallín Grande), región de Aysén, Chile, cuya autorización permitió conocer el estudio de las construcciones ubicadas dentro de sus predios, y por los testimonios de vida en torno de éstas. A la Universidad Austral de Chile Campus Patagonia, por facilitar el laboratorio y su equipamiento; y al arqueólogo Kemel Sade Martínez.

\section{Referencias}

Batista, Fabio

2011 A casa de madeira; um saber popular, Curitiba, Instituto Arquibrasil.

Bozzolo, Luis

1992 "Influencias en la arquitectura de Aisén", CA. Revista

Oficial del Colegio de Arquitectos de Chile s/n: 41-44.

Castillo, Carlos

2015a "Distribución geográfica de la arquitectura vernácula con tejuela artesanal, región de Aysén", Conserva 20: 7-21.

2015b "Locomóviles (motores a vapor) y la explotación maderera en la región de Aisén", Aysenología 0: 27-30.

Castillo, Crispín

2017 Comunicación personal, 20 de octubre, Ciudad de Coyhaique.

Castillo, Elica

2017 Comunicación personal, 10 de octubre, Ciudad de Coyhaique.

Dirección Meteorológica de Chile

2010 Descripción meteorológica, Undécima región, Documento técnico de la Dirección General de Aeronáutica Civil, Chile. 
Duncan, R. P.

1989 "An evaluation of errors in tree age estimates based on increment cores in Kahikatea (Dacrycarpus dacrydioides)", New Zealand Natural Sciences 16: 31-37.

Edensor, Tim

2016 "Incipient ruination: materiality, destructive agencies and repair", en Mikkel Bille y Tim Flohr Sørensen. Elements of Architecture. Assembling archaeology, atmosphere and the performance of building spaces, Londres, Nueva York, Routledge, pp. 348-364.

García Álvarez, Santos

2000 "La construcción de la vivienda tradicional en el Paraguay; la técnica del estaqueo", en A. Graciani et al., Actas del Tercer Congreso Nacional de Historia de la Construcción, Sevilla, 26-28 octubre de 2000, Madrid, Instituto Juan de Herrera, pp. 343-346.

Hajek, E., y F. di Castri

1975 "Bioclimatografía de Chile, manual de consulta", Dirección de Investigación Vice-Rectoría Académica Universidad Católica de Chile, Santiago, Chile.

Hepp, Cristian., y N. Stolpe

2014 "Caracterización y propiedades de los suelos de la Patagonia Occidental (Aysén)", Boletín del Instituto de Investigaciones Agropecuarias (INIA), 298.

Jorquera, Natalia

2015 "Aprendiendo del patrimonio vernáculo: tradición e innovación en el uso de la quincha en la Arquitectura Chilena", Revista de Arquitectura 28/29: 4-11.

Martinic, Mateo

2014 De la Trapananda al Aysén, Chile, Ediciones Fundación Río Baker.

Olivares Pinto, Luis (dir)

2006 Casas antiguas Coyhaique, Coyhaique, Gobierno Regional de Aysén / Cámara Chilena de la Construcción.

Osorio, Mauricio P.

2014 Antiguas historias del valle Simpson. Región de Aysén, Coyhaique, Ediciones Ñire Negro.

Pérez, Constanza, y C. Castillo

2016 Guía casas patrimoniales Coyhaique, Santiago, Andros Impresores.

Pérez, Lorena, T. Errázuriz y C. Castillo

2018 Casas en el valle Simpson, el patrimonio desconocido de Aysén, Santiago, Andros.

Proterra

s. f. "Quincha", documento electrónico disponible en [http://www.redproterra.org/subcategories/terminologia?per_ page $=270$ ], consultado en enero de 2019 .
Puchi, Paulina, A. Muñoz, M. González, A. Abarzúa, K. Araya, R. Towner, A. Fitzek, A. Holz y D. Stahle 2017 "Potencial de los anillos de crecimiento de Pilgerodendron uviferum para el estudio histórico de las iglesias de Chiloé", Patrimonio de la Humanidad, Bosque 38 (1): 109-121.

Urrutia, Elías 2018 Comunicación personal, 9 de septiembre, El Fraile-Coyhaique.

\section{Síntesis curricular del/los autor/es}

\section{Carlos Castillo Levicoy}

Investigador independiente, Chile

castillolevicoycarlos@gmail.com

Ingeniero forestal (Universidad Austral de Chile [UACH, Valdivia, Chile], magíster en ciencias con mención en botánica (Universidad de Concepción [UdeC], Chile). Actualmente, trabaja como consultor e investigador en el área forestal en la Región de Aysén, en Chile. Su trabajo de investigación durante los últimos años ha estado enfocado en la puesta en valor de los oficios tradicionales madereros y a estudiar la arquitectura vernácula regional, lo que le ha permitido publicar varios trabajos junto con otros profesionales. Es director y editor de la Revista de Aysenologia [www.aysenologia.cl]; socio-investigador de la Sociedad de Historia y Geografía de Aysén y de la ONG Aumen: el Eco de los Montes. Se desempeña como consultor e investigador en el área de bosques de la región de Aysén

\section{Constanza Pérez Lira}

Investigadora independiente, Chile

constanzaperezlira@gmail.com

Arquitecta (Pontificia Universidad Católica de Chile [PUC]), estudia un magíster de historia del arte (Universidad Adolfo Ibáñez, Santiago de Chile). Tras haber vivido cinco años en la región de Aysén, se ha dedicado al estudio y rescate del patrimonio cultural, contenida en la memoria, arquitectura y patrimonio fotográfico local de sus habitantes, en busca de una puesta en valor que constribuya al fortalecimiento de la identidad regional. 